\title{
Muscle strengthening of knee extensors in hemiparetics: prospective and longitudinal study
}

\author{
Wildja de Lima Gomes ${ }^{1}$, Thais Botossi Scalha² ${ }^{2}$ Ana Carolina Nunes Bovi ${ }^{3}$, Wagner de Mello e Castro4, \\ Amanda Pennacchi ${ }^{4}$, Gabriela Aparecida Gomes ${ }^{4}$, Mariana Taranto ${ }^{4}$, Enio Walker de Azevedo Cacho', \\ Núbia Maria Freire Vieira Lima'
}

\begin{abstract}
Background: Several attempts to reduce spasticity have been directed to stroke sufferers based on the historical view that spasticity is the major determinant of motor dysfunction and that its reduction results in improved function of the affected limb. Paresis is also recognized as a limiting factor in hemiparetic rehabilitation and has a negative impact on self-care, mobility, or up and down stairs due to slow activation of motor units and difficulty in producing adequate amount of muscle strength. In the treatment of spasticity, many therapeutic procedures have been used. Resisted exercises occur when there is load on the body segment distal to the muscle that develops muscle tension, occurs muscle shortening and an external force is overcome. Objective: The aim of this study was to analyze the results of isometric and isotonic strengthen program in the quadriceps muscles of the affected limb of patients with chronic hemiparesis secondary to stroke and to evaluate its effects on spasticity, motor function of the lower limb, balance and mobility. Methods: The participants were divided into three groups: isotonic strengthening, isometric strengthening and control group. It was applied the Ashworth scale, Fugl-Meyer Protocol, Time Up and Go test and Berg Balance Scale. Results: The isometric and isotonic strengthening program presented post-treatment variations, whereby only the isometry group showed improvement in balance. The control group did not show a significant improvement. There was no increase in the muscular tonus of the quadriceps or hamstrings immediately after the treatment. Conclusion: The selective muscle strengthening of the quadriceps resulted in an increase of the motor function, balance and mobility followed by a decrease or maintenance of the muscular tonus of the patients.
\end{abstract}

Keywords: Stroke; Muscular Spasticity; Paresis

\section{INTRODUCTION}

Several attempts to reduce spasticity have been directed to stroke sufferers based on the historical view that spasticity is the major determinant of motor dysfunction and that its reduction results in improved function of the affected limb $b^{(1)}$. Paresis is also recognized as a limiting factor in hemiparetic rehabilitation ${ }^{(2)}$ and has a negative impact on self-care, mobility, or up and down stairs due to slow activation of motor units and difficulty in producing adequate amount of muscle strength $^{(3,4)}$. Spasticity and synergies reduce the selectivity of hemiparetic movement and interfere with energy and efficiency in gait ${ }^{(5)}$. There is a correlation between muscle torque of knee extensors and gait velocity in hemiparetic ${ }^{(6)}$ and it is known that the spasticity of the ankle and knee muscles causes muscle co-activation and deceleration forces in the affected limb, which limits the step length in these patients ${ }^{(7)}$.

In the treatment of spasticity, many therapeutic procedures have been used, some of which have already been proven effective, such as pharmacological procedures, cryotherapy, passive mobilization, use of orthotic devices and neuromuscular electrical stimulation ${ }^{(8)}$ and others still enveloped in controversies, among them the muscle strengthening. Evidence that muscle strengthening increases muscle tone is weak ${ }^{(9)}$. Among the available strengthening options, there is isotonic training, which has been shown to be beneficial for this population and isometric training, which there are still few experimental studies published ${ }^{(8,10)}$. Isometric exercises are a static form of exercise in which the muscle contracts and produces force without an appreciable change in its length and without visible joint movement. Isotonic exercises are dynamic muscle contractions that cause joint movement and body segment excursion as the muscle contracts and shortens (concentric contraction) or stretches under tension (eccentric contraction). Resisted exercises occur when there is load on the body segment distal to the muscle that develops muscle tension, occurs muscle shortening and an external force (resistance) is overcome ${ }^{(11)}$. 
Normal motor performance requires execution of alternating motions at various functional speeds, with maintenance of appropriate temporal coordination between antagonistic muscles. Muscle training can reduce the amount of co-contraction, improve coordination and generation of selective force as spasticity can be minimized by gain of motor control. However, muscle strengthening programs have not been widely used in the rehabilitation of hemiparetics due to the fear of exacerbating the restriction imposed by spasticity and to reinforce the abnormal patterns of movement ${ }^{(12)}$. The objective of the study was to analyze the results of isometric and isotonic strengthening programs of the quadriceps musculature of the affected limb of patients with chronic hemiparesis secondary to stroke and to evaluate its effects on spasticity, motor function of the lower limb, balance and mobility.

\section{METHODS}

This is a prospective and longitudinal study carried out at the Physiotherapy and Occupational Therapy Outpatient Clinic of the Hospital das Clínicas of the UNICAMP, which was approved by the Research and Ethics Committee of the School of Health Sciences of the State University of Campinas (number 864/2008).

\section{Subjects}

Hemiparetic patients were recruited consecutively from a list of outpatient clinics. It was adopted as inclusion criterion patients with unilateral, ischemic or hemorrhagic stroke with more than 6 months of injury; grade IV or V in the quadriceps muscle function test of the affected limb. Exclusion criteria were patients with uncontrolled arterial hypertension; use of antispasmodic drugs or botulinum toxin in lower limb muscles; orthopedic (knee ankylosis) or neurological comorbidities; patients unable to understand the simple instructions and subjects who were participating in any other research. All subjects or guardians read and signed the informed consent form.

\section{Measure instruments}

For the categorization of the dysfunctional level of the patients the Functional Ambulation Scale (FAS) and Brunnstrom Rating were used for the affected lower extremity ${ }^{(13)}$. The FAS contains 5 criteria to evaluate bipedal walking ability, scoring from 0 to 5 , from an inability to ambulation, ambulation requiring supervision, to independent walking ${ }^{(14)}$. The Modified Ashworth Scale was used to assess the degree of spasticity of the knee extensor (quadriceps) and knee flexor (hamstring) muscles of the affected limb. The score of each item ranges from 0 to 4 , with 0 classified as normal tone and 4 as rigidity due to spasticity ${ }^{(15)}$. The Berg Balance Scale (BBS) measures the balance through 14 items and each is scored from 0 (worst possible performance) to 4 (normal performance) ${ }^{(16)}$.
The Fugl-Meyer Physical Performance Protocol (FM) evaluates the sensorimotor impairment of hemiparetic. The lower extremity motor function sections were used in the supine, sitting and standing positions and coordination / velocity of the lower extremity, with scores ranging from 0 (poor function) to 34 (normal function) ${ }^{(17)}$. In the Time up and Go test (TUG), the subjects were instructed to get up from a chair, walk for 3 meters, turn around, return to the chair and sit back on it. The time required to perform this test was measured by stopwatch ${ }^{(18)}$.

\section{Procedures}

The patients were randomly divided into three groups: the isometric muscle strengthening program group $(n=6)$, the isotonic muscle strengthening program group $(n=6)$ and the control group $(n=6)$. All patients participated in conventional physiotherapeutic treatment during the study period, at the frequency of 2 weekly sessions, based on neuroevolutionary techniques. The two strengthening groups participated in 12 sessions, these being 2 times a week with an average duration of 30 minutes. Blood pressure and heart rate were measured before and after the end of each session. In all treatment sessions the TUG Test was applied. The pre-treatment, post-treatment and follow-up evaluations were performed by the same professional, being a physiotherapist not involved with the treatment application. The isometric and isotonic strengthening groups were treated in the seated posture, with posterior support, hip and knee in position of $90^{\circ}$ and foot of unaffected limb supported (ankle in $90^{\circ}$ ).

Isometric Strengthening Group - The group treated with the maximal voluntary isometry technique performed the exercise sequence: (1) stretching of the quadriceps muscle of both lower limbs, with 3 repetitions of 30 seconds in maximal knee flexion, (2) (2) demonstration of the exercise in the lower limb not affected, and (3) muscle strengthening in 2 knee extension angles (30ㅇ and 60ㅇ, positioned through the goniometer), and concomitant percussion with hammer for reflexes in the active muscle, aiming to potentiate the contraction by means of the stretching reflex imposed. Three sets of 30 replicates were performed and the contraction sustained for 6 seconds in each replicate.

Concentric Isotonic Strengthening - This group performed an exercise program consisting of (1) quadriceps stretching of both lower limbs with 3 repetitions of 30 seconds in maximal knee flexion, (2) demonstration of exercise in the non-affected lower limb, and (3) muscle strengthening with load (shin guards) for the quadriceps muscle of the affected lower limb, with this training consisting of 3 sets of 30 repetitions, with 1 to 2 minutes' rest between sets. The amount of load that each participant used for the exercise was determined by the test of 10 maximum repetitions $-10 \mathrm{RMs}^{(19)}$. The maximum weight that the patient completed 10 movements was used as reference for the training, being performed series with $50 \%$ of that weight. In order to rebalance the load based 
on the participants' progress, this test was repeated at each session, by adding $1 / 2$ kilogram at each trial until the patient did not complete 10 movements. The test was performed in a maximum of 3 trials, with 2 minutes rest between them. The weights were readjusted to $50 \%$ of the new test result.

Control group - this group went through all initial and final evaluations, and underwent conventional physiotherapy (there was no muscle strengthening training).

The instruments of measurement were applied in the pre-treatment, post-treatment and follow-up phases (after 30 days of the end of the sessions). Only the control group was not evaluated in the follow-up phase.

\section{Statistical Analysis}

A descriptive analysis of the numerical and categorical variables of the groups was performed. There was no normal distribution of the variables by the Shapiro-Wilk test. The Friedman test was used to analyze the dependent variables of the same group and the Wilcoxon test to analyze two dependent variables of the same group. The Mann-Whitney test was used to correlate the variables between the 2 independent groups and the Kruskal-Wallis test to compare 3 independent groups. Values of $p<0.05$ were considered as statistically significant. Statistical software SPSS version 20.0 for Windows (IBM SPSS Statistics for Windows, Version 21.0, IBM Corp: Armonk, NY) was used.

\section{RESULTS}

The demographic characteristics of isometric, isotonic, and control muscle strengthening groups are shown in the table 1. There were no patients excluded in this study. Scores from each group on the BBS, TUG Test, Total FM and their sections for the 3 groups at pre-treatment, post-treatment and follow-up times are summarized in Tables 2, 3 and 4, respectively. No statistically significant differences were found between groups in relation to BBS post-treatment $(p=0.298)$, BBS follow-up ( $p=0.937)$, TUG post-treatment ( $p=0.229)$, TUG follow-up ( $p=0.937)$, FM post-treatment $(p=0.729)$ and total FM follow-up ( $p=0.589)$, as well as between sections of the FM protocol ( $p>0.05)$. Table 5 represents the distribution of quadriceps muscle tone degrees for the isometric, isotonic and control groups respectively.

\section{DISCUSSION}

The findings revealed that the isometric strengthening program presented better results in the motor function, balance and mobility of the chronic hemiparetic ones. There was no increase in muscle tone in the quadriceps or hamstrings immediately after training of both forms of muscle strengthening, but we found tonus elevation 30 days after the end of treatment. Recent evidence suggests that adaptive mechanisms of the musculoskeletal system, including an increase in passive muscle stiffness impairing the generation of force, are more directly associated with the motor dysfunctions
Table 1 - Characteristics of the groups of subjects in the pre-treatment evaluation.

\begin{tabular}{|c|c|c|c|}
\hline Variables & $\begin{array}{l}\text { Isometric } \\
\text { group }(n=6)\end{array}$ & $\begin{array}{c}\text { Isotonic } \\
\text { group }(n=6)\end{array}$ & $\begin{array}{l}\text { Control group } \\
\qquad(n=6)\end{array}$ \\
\hline Age (years) & $50.16 \pm 11.16$ & $48.16 \pm 13.99$ & $51.16 \pm 22.42$ \\
\hline Post-stroke time (years) & $4.5 \pm 1.87$ & $6.58 \pm 5.76$ & $4.33 \pm 1.96$ \\
\hline Gender (Male/Female) & $3 / 3$ & $3 / 3$ & $5 / 1$ \\
\hline Stroke type (H/I) & $2 / 4$ & $2 / 4$ & $2 / 4$ \\
\hline Hemiparesis side $(R / L)$ & $1 / 5$ & $4 / 2$ & $3 / 3$ \\
\hline Dominant side (R/L) & $5 / 1$ & $6 / 0$ & $3 / 3$ \\
\hline FAS & $4.33 \pm 0.81$ & $4 \pm 0.63$ & $3.5 \pm 1.37$ \\
\hline \multicolumn{4}{|l|}{$\begin{array}{l}\text { Classification of } \\
\text { Brunnstrom }\end{array}$} \\
\hline Stage I & -- & --- & -- \\
\hline Stage II & --- & --- & --- \\
\hline Stage III & 2 & 1 & 1 \\
\hline Stage IV & 2 & 2 & 2 \\
\hline Stage V & 2 & 3 & 3 \\
\hline Stage VI & -- & --- & -- \\
\hline
\end{tabular}

Note: H: hemorrhagic; I: ischemic; R: right; L: left; FAS: Functional Ambulation Scale.

Table $\mathbf{2}$ - Scores of the instruments of the Isotonic Group of measurement in the three periods

\begin{tabular}{cccrc}
\hline & \multicolumn{4}{c}{ Isotonic Group (n=6) } \\
\cline { 2 - 5 } Variables & Pre-treatment & $\begin{array}{c}\text { Post- } \\
\text { treatment }\end{array}$ & Follow-up & p-value \\
\cline { 2 - 5 } BBS & $49.16 \pm 4.16$ & $51.5 \pm 4.23$ & $51.83 \pm 4.16$ & 0.146 \\
TUG & $16.5 \pm 3.33$ & $11.66 \pm 2.8$ & $12 \pm 2.36$ & 0.006 \\
$\begin{array}{c}\text { Total FM - LL } \\
\text { Supine motor } \\
\text { function }\end{array}$ & $22 \pm 6$ & $25 \pm 6.22$ & $25.83 \pm 4.66$ & 0.001 \\
$\begin{array}{c}\text { Coordination/ } \\
\text { speed }\end{array}$ & $3.66 \pm 1.21$ & $3.5 \pm 1.04$ & $4.33 \pm 1.21$ & 0.292 \\
$\begin{array}{c}\text { Seated motor } \\
\text { function }\end{array}$ & $2.5 \pm 1.22$ & $2.66 \pm 1.36$ & $3.16 \pm 0.75$ & 0.097 \\
$\begin{array}{c}\text { Standing } \\
\text { motor function }\end{array}$ & $1.33 \pm 1.75$ & $1.83 \pm 1.72$ & $2 \pm 1.54$ & 0.039 \\
\hline
\end{tabular}

Note: BBS: Berg Balance Scale; TUG: Time up and go; FM: Fugl-Meyer Physical Performance Protocol; LL: lower limb.

presented by hemiparetic ones ${ }^{(20)}$. However, Junqueira et $a f^{(21)}$ did not observe a correlation between the increase in tonus and the gain of muscle strength. Teixeira-Salmela et al. ${ }^{(8)}$ demonstrated that muscle strengthening programs of the lower limb associated with aerobic training led to increased mobility, improved ladder performance, increased muscle strength without increased of tonus in the quadriceps and plantar flexors. I was not reevaluated the muscular strength, since the hemiparetic group should have at least degree IV of quadriceps strength to participate in the training. 
Table 3 - Scores of the measurement instruments of the Isometric group in the three periods.

\begin{tabular}{|c|c|c|c|c|}
\hline \multirow{2}{*}{ Variables } & \multicolumn{4}{|c|}{ Isometric Group $(n=6)$} \\
\hline & Pre-treatment & $\begin{array}{l}\text { Post- } \\
\text { treatment }\end{array}$ & Follow-up & p-value \\
\hline BBS & $49 \pm 5.17$ & $51.33 \pm 4.76$ & $52.16 \pm 3.97$ & 0.014 \\
\hline TUG & $16.33 \pm 5.12$ & $12.16 \pm 3.65$ & $12.5 \pm 4.13$ & 0.006 \\
\hline Total FM - LL & $21.66 \pm 5.57$ & $26.16 \pm 4.87$ & $27 \pm 4$ & $<0.001$ \\
\hline $\begin{array}{l}\text { Supine motor } \\
\text { function }\end{array}$ & $14.5 \pm 3.14$ & $16.83 \pm 2.48$ & $16.83 \pm 0.98$ & 0.174 \\
\hline $\begin{array}{l}\text { Coordination/ } \\
\text { speed }\end{array}$ & $4 \pm 1.09$ & $4.5 \pm 1.51$ & $5 \pm 0.89$ & 0.092 \\
\hline $\begin{array}{l}\text { Seated motor } \\
\text { function }\end{array}$ & $2 \pm 1.26$ & $3.16 \pm 0.98$ & $2.66 \pm 1.5$ & 0.047 \\
\hline $\begin{array}{l}\text { Standing } \\
\text { motor } \\
\text { function }\end{array}$ & $1.16 \pm 0.98$ & $1.66 \pm 1.63$ & $2.5 \pm 1.64$ & 0.015 \\
\hline
\end{tabular}

Note: BBS: Berg Balance Scale; TUG: Time up and go; FM: Fugl-Meyer Physical Performance Protocol; LL: lower limb.

Table 4 - Scores of the measurement instruments of the Control group in the two periods.

\begin{tabular}{lccc}
\hline \multirow{2}{*}{ Variables } & \multicolumn{3}{c}{ Control Group ( $\mathrm{n}=6$ ) } \\
\cline { 2 - 4 } & Pre-treatment & Post- treatment & p-value \\
\hline BBS & $41.66 \pm 9.04$ & $45.33 \pm 10.19$ & 0.072 \\
TUG & $17.5 \pm 8.4$ & $17.16 \pm 7.54$ & 0.577 \\
Total FM- LL & $21.3 \pm 4.27$ & $23.33 \pm 5.5$ & 0.068 \\
Supine motor function & $14.66 \pm 1.75$ & $16 \pm 1.41$ & 0.066 \\
Coordination/speed & $3.66 \pm 1.03$ & $3.66 \pm 1.75$ & 1 \\
Seated motor function & $2.33 \pm 1.21$ & $2.33 \pm 1.03$ & 1 \\
Standing motor & $0.66 \pm 0.81$ & $1.33 \pm 1.75$ & 0.194 \\
function &
\end{tabular}

Note: BBS: Berg Balance Scale; TUG: Time up and go; FM: Fugl-Meyer Physical Performance Protocol; LL: lower limb.

Muscle weakness is a common dysfunction in hemiparesis, and interventions that can improve muscle strength are an important part of post-stroke rehabilitation. Numerous studies indicate that resistance training programs have been well tolerated and provide increased muscle strength in hemiparetic ${ }^{(22,23)}$. Flansbjer et al. ${ }^{(24)}$ evaluated the effects of training with progressive resistance on muscle strength, muscle tonus, and gait performance using a dynamic and isokinetic muscle strengthening program. The results showed that the training improved the muscular strength in the lower limbs without provoking negative effects on the muscular tonus, in addition, the improvement of muscular strength was maintained at the follow-up, but did not correlate with the improvement of gait performance after intervention. The results corroborate with other authors who also indicated improvement of muscle strength in post-chronic stroke patients undergoing dynamic and isokinetic strengthening ${ }^{(25-26)}$.
Table 5 - Tonus degrees of knee extensors of the affected lower limb in the three periods.

\begin{tabular}{|c|c|c|c|}
\hline \multirow{2}{*}{ Patients } & \multicolumn{3}{|c|}{ Isometric group ( $n=6)$} \\
\hline & Pre-treatment & Post-treatment & Follow-up \\
\hline 1 & 0 & 0 & +1 \\
\hline 2 & 2 & 2 & +1 \\
\hline 3 & 1 & 1 & +1 \\
\hline 4 & 0 & 0 & 1 \\
\hline 5 & 1 & 0 & 0 \\
\hline \multirow[t]{3}{*}{6} & 1 & 0 & 0 \\
\hline & \multicolumn{3}{|c|}{ Isotonic group $(n=6)$} \\
\hline & Pre-treatment & Post-treatment & Follow-up \\
\hline 1 & 0 & 0 & +1 \\
\hline 2 & 1 & 0 & 1 \\
\hline 3 & 0 & 0 & 1 \\
\hline 4 & 1 & 0 & 0 \\
\hline 5 & 1 & 1 & 0 \\
\hline \multirow[t]{3}{*}{6} & 0 & 0 & +1 \\
\hline & \multicolumn{3}{|c|}{ Control group $(n=6)$} \\
\hline & \multicolumn{2}{|c|}{ Pre-treatment } & Post-treatment \\
\hline 1 & \multicolumn{2}{|c|}{1} & 1 \\
\hline 2 & \multicolumn{2}{|c|}{1} & 0 \\
\hline 3 & \multicolumn{2}{|c|}{1} & 0 \\
\hline 4 & \multicolumn{2}{|c|}{+1} & 1 \\
\hline 5 & \multicolumn{2}{|c|}{1} & +1 \\
\hline 6 & \multicolumn{2}{|c|}{0} & 0 \\
\hline
\end{tabular}

Sharp et al. (2) developed a program of isokinetic strengthening of the quadriceps and hamstring muscles of hemiparetics subjects for 6 weeks. The authors found significant gains in muscle strength after training without increased tonus and increased gait velocity, however TUG results did not change with statistical significance. The authors reported that post-strengthening selective muscle changes may not be sufficient to improve performance on complex tasks such as mobility and gait. However, we found significant variations for TUG in both strengthening groups. Maynard et al. ${ }^{(5)}$ developed a training program for hemiparetic quadriceps and plantar flexors, which consisted of a single session of twenty minutes of isokinetic or isotonic exercises associated or not with weight loss. Hemiparetics submitted to isotonic exercises without weight loss demonstrated an increase in walking speed. Our findings revealed reduced mobility time by the TUG test in both training groups. Ryan et al ${ }^{(27)}$ proposed a resistance training protocol for a period of 3 months (with sessions 3 times a week). The program showed hypertrophy in the paretic lower limb and decreased intramuscular fat. The results showed no change in gait velocity after rehabilitation, but it 
was observed that patients were able to travel longer distances when compared to the control group. Other authors ${ }^{(28-29)}$ also showed an increase in walking speed, in the length of the step, and in the distance walked in the 6-minute walk test from a resistance exercise protocol.

Teixeira-Salmela et al. ${ }^{(3)}$ trained hemiparetics subjects through weight training for lower limbs, upper limbs and trunk and aerobic conditioning and proposed the weight training as a safe and viable way for their rehabilitation. The authors pointed out that the training of isolated muscle groups, such as knee flexors and extensors, may not be sufficient to generate gains in more complex motor tasks. However, it was found optimization of mobility (set of tasks: lifting, walking, rotating and sitting) after strengthening the quadriceps, which can be explained by the improvement of lower limb function. Similarly Jørgensen et al ${ }^{(30)}$ applied a resisted exercise protocol associated with aerobic exercises. The results pointed to the improvement of the gait performance, evaluated from the 6-minute walk test. Ouellette et al. ${ }^{(10)}$ demonstrated that a bilateral training program with knee extension, dorsiflexion and ankle plantar flexion can improve muscle strength and power in both lower extremities of the paretic and non-paretic side. This improvement is associated with increased lower extremity function and reduced spasticity in chronic hemiparetic subjects. In our study, the reduction in spasticity seen in hamstring muscles may be due to medullary reflex activity by reciprocal inhibition.

\section{CONCLUSION}

Selective quadriceps muscle strengthening, especially isometric training, resulted in improved motor function, balance and mobility accompanied by reduction or maintenance of muscle tonus in most patients. Thus, strengthening was shown to be an effective program in the treatment of mobility and walking deficits of post-stroke hemiparetic patients in a chronic stage.

\section{AUTHORS' CONTRIBUTIONS}

WLG: contributed to the intellectual content of this work, according to the ICMJE guidelines, in the critical review, revising the intellectual content of the manuscript before the final presentation. TBS and ACNB: contributed to the intellectual content of this work, according to the ICMJE guidelines, in the data collection and treatment, literature survey and analysis/interpretation. WMC, AP and GAG: contributed to the intellectual content of this work, according to the ICMJE guidelines, in the data collection and treatment and writing of the manuscript. MT: contributed to the intellectual content of this work, according to the ICMJE guidelines, in the literature survey and analysis/ interpretation. EWAC and NMFVL contributed to the intellectual content of this work, according to the ICMJE guidelines, in design and development, methodological design and critical review.

\section{CONFLICTS OF INTEREST}

The authors declare that there was no conflict of interests.

\section{AUTHOR DETAILS}

${ }^{2}$ University of Sorocaba (UNISO), Sorocaba- SP, Brazil. ${ }^{3}$ University of Sorocaba (UNISO), São Paulo-SP, Brazil. ${ }^{4}$ University of Campinas (UNICAMP) Campinas-SP, Brazil

\section{REFERENCES}

1. O'Dwyer N, Ada L, Neilson P. Spasticity and muscle contracture following stroke. Brain. 1996;119(5):1737-1749

2. Sharp S, Brouwer B. Isokinetic strength training of the hemiparetic knee: Effects on function and spasticity. Archives of Physical Medicine and Rehabilitation. 1997;78(11):1231-1236

3. Teixeira-Salmela L, Oliveira E, Santana E, Resende G. Muscle strengthening and physical conditioning in chronic stroke subjects. 2017.

4. Teixeira-Salmela L, Silva P, Lima R, Augusto A, Souza A, Goulart F. Exercise Machines and aerobic conditioning on functional performance of chronic stroke survivors. Acta Fisiátrica. 2003;10(2).

5. Maynard V, Bakheit A, Shaw S. Comparison of the impact of a single session of isokinetic or isotonic muscle stretch on gait in patients with spastic hemiparesis. Clinical Rehabilitation. 2005;19(2):146-154.

6. Lindmark B, Hamrin E. Relation between Gait Speed, Knee Muscle Torque and Motor Scores in Post-Stroke Patients. Scandinavian Journal of Caring Sciences. 1995;9(4):195-202.

7. Corrêa F, Soares F, Andrade D, Gondo R, Peres J, Fernandes A et al. Atividade muscular durante a marcha após acidente vascular encefálico. Arquivos de Neuro-Psiquiatria. 2005;63(3b):847-851.

8. Teixeira-Salmela L, Olney S, Nadeau S, Brouwer B. Muscle strengthening and physical conditioning to reduce impairment and disability in chronic stroke survivors. Archives of Physical Medicine and Rehabilitation. 1999;80(10):1211-1218.

9. Morris S, Dodd K, Morris M. Outcomes of progressive resistance strength training following stroke: a systematic review. Clinical Rehabilitation. 2004;18(1):27-39.

10. Ouellette M, LeBrasseur N, Bean J, Phillips E, Stein J, Frontera W et al. High-Intensity Resistance Training Improves Muscle Strength, SelfReported Function, and Disability in Long-Term Stroke Survivors. Stroke. 2004;35(6):1404-1409

11. Kisner C, Colby L. Exercicios terapeuticos. Barueri (SP): Manole; 2009.

12. Holden M, Gill K, Magliozzi M. Gait Assessment for Neurologically Impaired Patients. Physical Therapy. 1986;66(10):1530-1539.

13. Brunnstrom S. Motor testing procedures in hemiplegia: based on sequential recovery stages. Phys Ther. 1966 Apr;46(4):357-75.

14. Jonsson M, Tollbäck A, Gonzales H, Borg J. Inter-rater reliability of the 1992 international standards for neurological and functional classification of incomplete spinal cord injury. Spinal Cord. 2000;38(11):675-679

15. Miyamoto S, Lombardi Junior I, Berg K, Ramos L, Natour J. Brazilian version of the Berg balance scale. Brazilian Journal of Medical and Biological Research. 2004;37(9):1411-1421.

16. Maki T, Quagliato E, Cacho E, Paz L, Nascimento N, Inoue M et al. Estudo de confiabilidade da aplicação da escala de Fugl-Meyer no Brasil. Revista Brasileira de Fisioterapia. 2006;10(2):177-183

17. Ng S, Hui-Chan C. The Timed Up \& Go Test: Its Reliability and Association With Lower-Limb Impairments and Locomotor Capacities in People With Chronic Stroke. Archives of Physical Medicine and Rehabilitation. 2005;86(8):1641-1647.

18. Aveiro M, Granito R, Navega M, Driusso P, Oishi J. Influence of a physical training program on muscle strength, balance and gait velocity among women with osteoporosis. Revista Brasileira de Fisioterapia. 2006;10(4).

19. Lee M, Kilbreath S, Singh M, Zeman B, Lord S, Raymond J et al. Comparison of Effect of Aerobic Cycle Training and Progressive Resistance Training on Walking Ability After Stroke: A Randomized Sham Exerciseâ€“Controlled Study. Journal of the American Geriatrics Society. 2008;56(6):976-985.

20. Moraes, GFS; Nascimento, LR; Glória, AE; Teixeira-Salmel, LF; Paiva, CMP; Lopes, TAT et al. The influence of muscle strengthening on upper limb motor performance in stroke subjects. Acta Fisiátr.2008;15(4):245-248.

21. Junqueira, RT, Ribeiro, AM and Sciann, AA. "Efeitos do fortalecimento muscular e sua relação com a atividade funcional e a espasticidade em indivíduos hemiparéticos." Braz. j. phys. ther.(Impr.) 2004: (8.3) 247-252. 
22. Lee M, Kilbreath S, Singh M, Zeman B, Lord S, Raymond J et al. Comparison of Effect of Aerobic Cycle Training and Progressive Resistance Training on Walking Ability After Stroke: A Randomized Sham Exerciseâ€“Controlled Study. Journal of the American Geriatrics Society. 2008;56(6):976-985.

23. Lee M, Kilbreath S, Singh M, Zeman B, Davis G. Effect of Progressive Resistance Training on Muscle Performance after Chronic Stroke. Medicine \& Science in Sports \& Exercise. 2010;42(1):23-34.

24. Flansbjer $U$, Miller $M$, Downham D, Lexell J. Progressive resistance training after stroke: Effects on muscle strength, muscle tone, gait performance and perceived participation. Journal of Rehabilitation Medicine. 2008;40(1):42-48.

25. Lee S, Kang K. The Effects of Isokinetic Eccentric Resistance Exercise for the Hip Joint on Functional Gait of Stroke Patients. Journal of Physical Therapy Science. 2013;25(9):1177-1179.

26. Badics, E., Wittmann, A., Rupp, M., Stabauer, B., \& Zifko, U. A. Systematic muscle building exercises in the rehabilitation of stroke patients." NeuroRehabilitation 17.3 (2002): 211-214
27. Ryan A, Ivey F, Prior S, Li G, Hafer-Macko C. Skeletal Muscle Hypertrophy and Muscle Myostatin Reduction After Resistive Training in Stroke Survivors. Stroke. 2010;42(2):416-420.

28. Bale M, Inger Strand L. Does functional strength training of the leg in subacute stroke improve physical performance? A pilot randomized controlled trial. Clinical Rehabilitation. 2008;22(10-11):911-921.

29. Cooke E, Tallis R, Clark A, Pomeroy V. Efficacy of Functional Strength Training on Restoration of Lower-Limb Motor Function Early After Stroke: Phase I Randomized Controlled Trial. Neurorehabilitation and Neural Repair. 2009;24(1):88-96.

30. Jorgensen J, Bech-Pedersen D, Zeeman P, Sorensen J, Andersen L, Schonberger M. Effect of Intensive Outpatient Physical Training on Gait Performance and Cardiovascular Health in People With Hemiparesis After Stroke. Physical Therapy. 2010;90(4):527-537. 